$\underline{\text { Preprint typeset in JHEP style. - HYPER VERSION }}$

MIT-CTP-2946

TAUP-2609-2000

NSF-ITP-00-07

hep-th/0003025

\title{
On Orientifolds, Discrete Torsion, Branes and $M$ Theory
}

\author{
Amihay Hanany \\ Center for Theoretical Physics, \\ Massachusetts Institute of Technology \\ Cambridge MA 02139 \\ hanany@mit.edu \\ Barak Kol \\ School of Physics and Astronomy \\ Tel Aviv University \\ Ramat Aviv 69978, Israel \\ barak1k@post.tau.ac.il
}

\begin{abstract}
We find some lifts to $M$ theory of orientifold and orbifold planes including the O1, O3 and O5 planes of Type IIB and their transformations under $S L(2, \mathbf{Z})$. The possible discrete torsion variants (or $\mathrm{K}$ theory classes) are explored, and are interpreted as arising from brane intersections with planes. We find new variants of the $\mathrm{O} 0$ and of an orbifold line (OF1) and determine their tensions in some cases. A systematic review of orientifolds, $\mathrm{M}$ orientifolds, and known $\mathrm{M}$ lifts, with some new clarifications is included together with a discussion of the role of $\mathrm{T}$ duality.
\end{abstract}

KEYwords: M-theory, p-branes. 


\section{Contents}

1. Introduction 2

2. Introduction to Orientifolds

2.1 Brane Realization of Discrete Torsion 6

2.2 T Duality 8

3. Review of M-lifts of Orientifolds 9

3.1 The OM5 plane 10

3.1.1 M-lift of the $\mathrm{O} 4$

3.2 The OM2 plane 12

3.2.1 The brane - orientifold intersection in M theory 12

3.2 .2 M-lift of the $\mathrm{O} 2$

3.3 The OM1 line 13

4. M lifts of Type IIB Orientifolds 14

4.1 O3 14

4.2 O5 - ON5

4.3 O1 - OF1 17

5. Orbifolds, Orientifolds and New Variants 18

5.1 Orbifold lines 18

5.2 Orbifold 5 planes 21

5.3 New variants of the O0 plane 22

6. Miscellaneous applications 22

6.1 The spectrum of $4 \mathrm{~d} \mathcal{N}=4$ with $S O, S p$ gauge group 22

6.2 A comment on allowed BPS stated and $\mathbf{Z}_{2}$ charges 23

6.3 Orientifold webs 24

A. Appendix - Cohomology of RP 25 


\section{Introduction}

Orientifold planes are objects in string theory which are defined perturbatively by gauging a discrete symmetry which involves reversing the sign of coordinates transverse to the plane while changing the orientation of the string [1, 2, 3]. Before we delve into the details of their construction and their dynamics let us mention some motivation and interest in their study.

Orientifold planes are of interest in many aspects of string theory. They turn out to be useful in studying disconnected components in moduli space of various string compactifications. They are crucial in the brane construction of gauge theories with $S p / S O$ gauge groups. ${ }^{1}$ In addition they give a simple realization of matter in symmetric and antisymmetric second rank representations in $S U(N)$ gauge theories.

As will be discussed in detail in the following sections, orientifold planes turn out to be characterized by discrete fluxes. Some of these fluxes originate from NS fields (See [5] for a recent discussion) and some other fluxes come from RR fields. While the first (NS) are visible from the usual perturbative description of orientifolds, the latter (RR) cannot be treated in a perturbative formulation of the Type II string since, as of now, there is no formalism which includes RR backgrounds in string theory. For this reason we need to rely on other methods in order to study such objects, and once such a formalism is available, these questions should be revisited. The best analysis which is available at the moment will include essentially two tools - various dualities, and discrete fluxes represented by intersections of branes with the orientifold plane.

Here we study two subjects related to orientifolds - their lift to M theory (See a recent related discussion in [6]), and the different variants they appear in. We begin with a review of the relevant features of orientifolds in section 2. We describe the 4 types of $O p$ planes which are known to exist for $p \leq 5$. We explain how these 4 types are classified by a pair of $\mathbf{Z}_{2}$ parameters, one perturbative coming from the $B_{N S}$ form and the other from one of the RR forms. These parameters are "discrete torsion" parameters which arise whenever the field strength of a p-form potential has a corresponding non trivial discrete cohomology in space time [7, 8] (see also recent related work 19, 10, 11, 12]). Equivalently we show that these variants arise as brane configurations in the spirit of [13] (see recent related work [14]) from intersections of an O plane with a brane which divides it into two parts. We search for all the possible discrete torsion variants, and we find that for $p \leq 1$ there are some additional ones. Later in that section, in 2.2, we explain how $\mathrm{T}$ duality relates a wrapped orientifold with a pair of lower dimensional orientifolds and the mapping between the various discrete torsion variants.

\footnotetext{
${ }^{1}$ See $[4$ for a recent interesting application.
} 
In section 3 we systematically review the $\mathbf{Z}_{2}$ orbifolds of $\mathbf{M}$ theory. There are only a few of these and upon compactification they give rise to the various $\mathbf{Z}_{2}$ orientifolds and orbifolds of string theory. We review the M lifts of the O0, O2, O4 [15, 16, 17, 18, 19, 20, 21 in terms of 11d O planes - the OM1, OM2 and OM5. In the case of the O2 we give a new $\mathrm{M}$ theory explanation for the difference between the two variants $\mathrm{O}_{2}^{+}$ and $\widetilde{O 2}^{+}$.

In section 4 we describe the $\mathrm{M}$ lift of the $\mathrm{O} 1, \mathrm{O} 3$ and $\mathrm{O} 5$ in terms of the same OM planes. In all cases we pay special attention to the $S L(2, \mathbf{Z})$ symmetry [22] (see also related work 23]). For the $\mathrm{O} 3$ we show how the $\mathrm{M}$ lift realizes the $S L(2, \mathbf{Z})$ geometrically, as usual. For the $\mathrm{O} 1$ and $\mathrm{O} 5$ the $\mathrm{S}$ transformation of $S L(2, \mathbf{Z})$ transforms the orientifold planes into orbifolds, which originate in the same OM planes.

In section 5 we discuss in detail the interrelations between $O p$ planes and orbifolds for $p=1,5$. We explore the possible discrete torsion variants and discuss new ones for the orbifold and orientifold lines and for the orientifold point. We compare the discrete torsion classification with a recent claim on a $\mathrm{K}$ theory classification [24] (see 25, 26, 27, 28, 29, 30 for earlier and related work) and find groups of the same order but with a different group structure. We are able to find the tension of some of the variants, and it remains to do so for the other cases.

Finally we discuss some miscellaneous applications in section 6. We discuss the dyon spectrum for $4 \mathrm{~d} \mathcal{N}=4$ with $S O / S p$ gauge groups, where the $S p$ theory has two versions. Then the discussion on the monopole spectrum of such a theory is generalized to a field theory in an arbitrary dimension. Finally, we discuss the problems associated with a $(p, q)$ web of orientifolds.

Let us mention some open questions

- We show how an intersection of an orientifold, or an OM plane, with a brane causes a tension jump, and so a fractional charge is deposited on the brane. This jump should be understood and probably required by the worldvolume theory on the brane. In particular consistency with Dirac quantization is required.

- We find the tension of some of the discrete torsion variants of the orbifold lines, and it would be interesting to know all of them.

Preliminary results of this work were presented in Santa Barbara and can be found in the following link: http://online.itp.ucsb.edu/online/susy99/hanany/. 


\section{Introduction to Orientifolds}

An orientifold plane in $p+1$ dimensions is defined as Type II string theory on $R^{p, 1} \times$ $R^{9-p} /(I \cdot \Omega \cdot J)$ where $I$ is the inversion of all coordinates in the transverse space $R^{9-p}$, $\Omega$ is the orientation reversal on the world sheet of the fundamental string and $J$ is the identity operator for $p=0,1 \bmod 4$ and $(-1)^{F_{L}}$, the left moving spacetime fermion number operator, for $p=2,3 \bmod 4$ [31]. We will denote an orientifold p-plane as an "Op plane".

By definition the orientifold acts on $B_{N S}$ (with components parallel to the $O p$ plane) with a sign reversal. The action on the other NS fields is trivial, so let us specify the action on all of the RR forms. The $O 9$ which projects Type IIB to Type I keeps invariant the left-right symmetric part of the spectrum, which in the RR sector is the 2 -form $B_{R R}$. By $\mathrm{T}$ dualizing we arrive at the following rule for the action of an $O p$ plane on the RR forms $C_{p^{\prime}}$

$$
\begin{aligned}
C_{p^{\prime}} \rightarrow C_{p^{\prime}} & p^{\prime}=p+1 \bmod 4 \\
C_{p^{\prime}} \rightarrow-C_{p^{\prime}} & p^{\prime}=p+3 \bmod 4 .
\end{aligned}
$$

Note that the above sign comes in addition to a component dependent sign which comes from the tensor transformation rules. Forms which get reversed by the action of the group are termed "twisted", and this notation should not be confused with forms from a twisted sector.

The transverse space to an $O p$ plane is an $\mathbf{R P}^{\mathbf{8}-\mathbf{p}}$. This space has some (discrete) torsion cohomologies which are summarized in appendix A. Whenever a space has discrete torsion, forms of an appropriate rank are topologically classified by it. Namely

$$
\left[G_{p+1}\right] \in H^{p+1}(X, \mathbf{Z})
$$

where $H^{p+1}(X, Z)$ is the integral cohomology of the space $X$ and $G_{p+1}$ is the RR field strength $p+1$-form. Twisted forms require the use of twisted cohomologies, $\tilde{H}^{p+1}$. In the case of orientifolds we will see that discrete torsion allows for the orientifold to appear in several variants.

For a general orientifolds $(p \leq 5)$ there are at least four types of orientifold planes which are distinguished by two $\mathbf{Z}_{2}$ charges. One $\mathbf{Z}_{2}$ charge comes from the NS sector and the other comes from the RR sector. For this reason, the former charge is seen in perturbative string theory while the latter is not. The perturbative distinction was first discussed in modern language in [3]. Later, the other $\mathbf{Z}_{2}$ charge was discovered in $[32 \text {, 33 }]^{2}$ for the case $p=3$ essentially by using field theory intuition which is based on

\footnotetext{
${ }^{2}$ In 34 there is an earlier discussion of the four variants of 5 planes in an M theory language which is directly related to discrete torsions.
} 
Montonen Olive Duality or Type IIB self duality. The case $p=4$ was studied in detail by [20, 21].

For an $O p$ plane, the two $\mathbf{Z}_{2}$ charges are given by $b$, which is the class $\left[d B_{N S}\right]=$ $\left[H_{N S}\right] \in \tilde{H}^{3}\left(\mathbf{R P}^{\mathbf{8}-\mathbf{p}}\right)$ and by $c$, which is the class of an $\mathrm{RR}$ form $\left[d C_{5-p}\right]=\left[G_{6-p}\right] \in \mathbf{Z}_{2}$ (the relevant cohomology, either twisted or untwisted, is found to be $\mathbf{Z}_{2}$ ). In the next subsection we will give these a physical interpretation via brane-orientifold intersections. The above classes can be defined for $p \leq 5$, while for $6 \leq p \leq 8$ one can still define $b$ through the perturbative action on open strings. In general the existence of a discrete torsion implies the existence of variants, though variants could possibly exist without it, as in the case of the $b$ variant. The absence of a $c$ charge for $p=6$ suggests that, contrary to naive expectations, one can not have an $\widetilde{O 6}^{-}$plane, i.e. half a D6 brane stuck on an $O 6^{-}$plane. That turns out to be the case as implied by the results of [35].

One can systematically check for any $p$ which additional forms can get discrete torsions. For low $p$ some extra variants exist (see section 5): for $p \leq 1$ one can define an additional charge $c^{\prime}$ by $\left[d C_{1-p}\right]=\left[G_{2-p}\right] \in \mathbf{Z}_{2}$, and for $p=1$ one has $* H_{N S} \in \tilde{H}^{7}\left(\mathbf{R P}^{\mathbf{7}}\right)=\mathbf{Z}_{\mathbf{2}}$. In addition there are two other discrete torsion whose variants we ignore $-H^{0}\left(\mathbf{R P}^{\mathbf{n}}\right)=\mathbf{Z}$ leads to a integral discrete variant for $p=2 \bmod 4$ which we interpret as describing the possible massive theories of type IIA (this interpretation is based on the brane intersection picture, where this torsion is seen to be due to an intersection with a D8 plane). The other type which we ignore is $H^{8-p}=\mathbf{Z}$ for odd $p$ and $\tilde{H}^{8-p}=\mathbf{Z}$ for even $p$, which simply means that one can add to an $O p$ plane any integer number of $D p$ branes.

The orientifold planes will be denoted according to their $\mathbf{Z}_{2}$ charges. A trivial $b$ charge will be denoted by a ${ }^{-}$superscript and a non-trivial $b$ will be denoted by + . A non-trivial $c$ charge will be denoted by adding $\mathrm{a}^{\sim}$. The charges of these planes are $-2^{p-5}$ for the $O p^{-}$plane, $+2^{p-5}$ for both the $O p^{+}$and the $\widetilde{O p^{+}}$and $\frac{1}{2}-2^{p-5}$ for the $\widetilde{O p^{-}}$plane. The tensions of these objects are measured in units of the Dp brane tension and are identical to their charge. Note a change of notation for the various orientifold planes from the papers [36, 16]. The orientifold planes are denoted there $O p^{-}, O p^{+}, \widehat{O p}$, and $\widetilde{O p}$, respectively. The new notation is based on the two $\mathbf{Z}_{2}$ charges and makes it more simple to work with. $n$ physical $D p$ branes stacked upon an $O p$ plane leads to a gauge group $S O(2 n), S p(n), S O(2 n+1)$ and $S p(n)$ for $O p^{-}, O p^{+}, \widetilde{O p^{-}}, \widetilde{O p^{+}}$, respectively. The two $S p(n)$ theories differ, in some cases, by a theta angle and in their monopole spectrum. This will be discussed in detail in sections 6.1 and 6.2. 


\subsection{Brane Realization of Discrete Torsion}

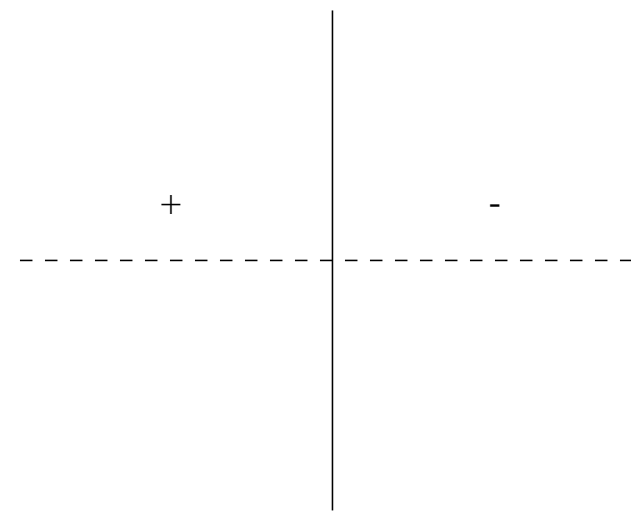

Figure 1: A half NS brane and an Orientifold plane. The solid line represents a half NS brane and the dashed line represents an $O p$ plane. The type of the $O p$ plane changes from $\mathrm{Op}^{-}$to $\mathrm{Op}^{+}$as it crosses the half NS brane.

In this section we will use a basic fact about orientifold planes and NS five branes and will develop a set of relations between intersecting branes and orientifold planes. The basic observation, made in [37, can be described in figure 1. Consider a NS five brane which spans the 012345 coordinates and intersecting an $O p$ plane which spans the $0, \ldots, p-1$, and 6 coordinates, $p \leq 6$. It splits the orientifold plane into two different parts, one to its left and one to its right and the orientifold plane changes its type as in figure 1.

The NS brane in this configuration has the special property that it is reflected onto itself by the orientifold. In a compact configuration such a brane would have half of the charge of a brane which does not intersect the orientifold and so we call it a $\frac{1}{2}$ NS brane. Note that a $1 / 2$ brane which extends outside of the orientifold has the same charge density as a unit brane, only it covers half of the volume due to the projection. On the other hand, a half brane inside the orientifold has half the charge density. A 1/2 brane cannot move in directions transverse to the orientifold plane, because only integral branes can move away off the orientifold. In the following brane configurations one should bear in mind that all branes which intersect with an orientifold plane are 1/2 branes.

Let us emphasize this point in more detail. Consider a configuration in which a physical NS brane is located far away from the orientifold, as in figure 2a. Let this brane move slowly towards the orientifold plane. It moves together with its image under the orientifold projection. As the two images meet on the orientifold plane, they can split along it as in figure 2 $\mathrm{b}$. At this point the rule in figure 1 can be used and a new type of orientifold plane is generated in between the two half NS branes.

We can look at it from a different point of view starting from figure 2 $2 \mathrm{~b}$ and moving to figure 2 a. As observed in [37, the type of the orientifold plane changes as one crosses the $\frac{1}{2}$ NS brane. This is determined by the $b$ charge of the orientifold which changes as one crosses the $\frac{1}{2}$ NS brane. On the other hand, if we add an additional $\frac{1}{2}$ NS brane, the type of the orientifold changes back to its original value, as in figure $2 \mathrm{~b}$. In this case we can have a dynamical process in which the two half NS branes combine together along the 6 coordinate and leave the orientifold along the 789 directions as in figure 2 a. 

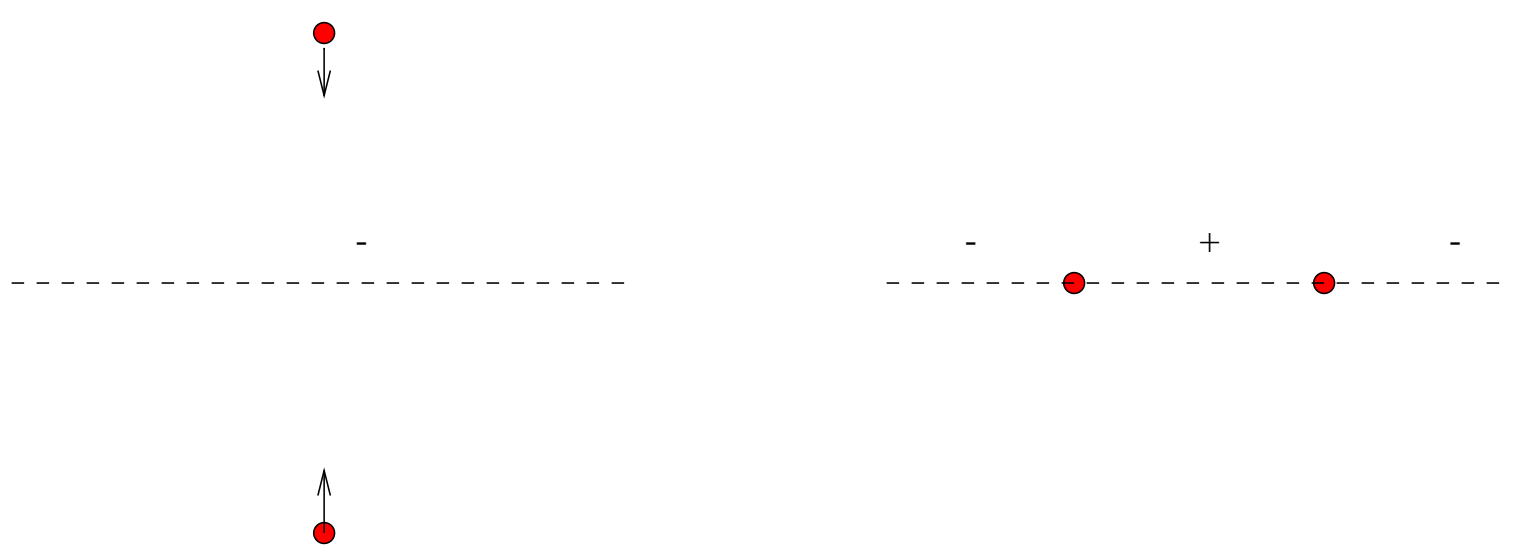

Figure 2: A physical NS brane (and its image) near an orientifold plane. In the left figure (a), the NS brane and its image are away from the orientifold plane. As they move and meet along the $O p$ plane they can split into a pair of half NS branes and form a new type of orientifold plane (b). The $O p$ types are denoted by + and - signs.

This is the inverse process to the one described in the last paragraph.

One can relate such configurations to the value of the two form flux $b$ by the following reasoning. The combined configuration of a $D p$ brane and half an NS brane is located at a point in 789 directions. There is a corresponding $\mathbf{R P}^{\mathbf{2}}$ which surrounds the configuration. The field which couples magnetically to the NS brane is the 2 -form NS field. Consequently, the integral of the two form over the $\mathbf{R P}^{\mathbf{2}}$ measures the number of $1 / 2$ NS branes, mod 2, which are located within the $\mathbf{R P}^{\mathbf{2}}{ }^{3}$.

$$
\exp \left(i \int_{\mathbf{R P}^{2}} B_{N S}\right)=(-)^{\# \frac{1}{2} \mathrm{NS} \text { branes }}
$$

This holonomy ("Wilson loop") $\in H^{p}(X, U(1))$ of a general p-form potential has the same discrete torsion as the field strength class $\in H^{p+1}(X, Z)$ which was mentioned before. This is seen via the exact sequences $0 \rightarrow \mathbf{Z} \rightarrow \mathbf{R} \rightarrow U(1) \rightarrow 0$ and

$$
\begin{aligned}
\ldots & \rightarrow H^{p}(X, \mathbf{R}) \rightarrow H^{p}(X, U(1)) \rightarrow \\
\rightarrow H^{p+1}(X, \mathbf{Z}) & \rightarrow H^{p+1}(X, \mathbf{R}) \rightarrow \ldots
\end{aligned}
$$

where the cohomologies with real coefficients do not have torsion parts.

We will assume that the configuration of figure 1 exists and will apply some $\mathrm{S}$ and $\mathrm{T}$ dualities to see what other results can be obtained from this basic configuration. First let us apply $\mathrm{S}$ duality to this configuration in the case $p=3$ (see also [39]). For this we need to know what are the $\mathrm{S}$ transformations of the various orientifold planes.

\footnotetext{
${ }^{3} \mathrm{~A}$ discussion in a similar spirit can be found in [38].
} 
This is easily done by looking at their $\mathbf{Z}_{2}$ charges which are the NS 3-form and the RR 3 -form field strengths. Let us recall that $O 3^{-}$has charge $(b, c)=(0,0)$ and the $\widetilde{\mathrm{O3}^{+}}$ has charge $(1,1)$. These orientifolds are self-dual under $\mathrm{S}$ duality. $O 3^{+}$has $(1,0)$ and transforms under $\mathrm{S}$ duality to $(0,1)$ which is the $\widetilde{\mathrm{O3}^{-}}$.

Equipped with this data let us make an $\mathrm{S}$ duality on the configuration of figure

17. One gets that an $\mathrm{O3}^{-}$transforms to an $\widetilde{\mathrm{O3}^{-}}$while crossing a half D5 brane. By crossing another half NS brane the $b$ charge of the orientifold jumps and one gets $\widetilde{\mathrm{O3}^{+}}$. This is expected if one assumes that the configuration of half D5 and half NS brane are self dual under S duality and that the order of the D5 and NS brane is not important. The summary is that when crossing a half NS brane the $b$ charge changes while when crossing a half D5 brane the $c$ charge of the orientifold changes. $b$ measures the number of half NS branes enclosed by an $\mathbf{R P}^{2}$ surrounding the intersection of O3 plane and NS brane while $c$ measures the number of half D5 branes enclosed by an $\mathbf{R P}^{\mathbf{2}}$ surrounding the intersection of O3 plane and D5 brane.

Next we can perform $\mathrm{T}$ duality on these results. Consider dualizing this system in a supersymmetric fashion to a system of a NS brane along 012345 and a D6 brane along 0123789. The orientifold plane dualizes to an O4 plane along 01236. The two $\mathbf{Z}_{2}$ charges are measured by $b$ and by $c$ which is a Wilson line of the RR one form of Type IIA. T duality then implies that when an O4 plane crosses a half NS brane its $b$ charge changes while when crossing a half D6 brane its $c$ charge changes (A Wilson line $c$ can be associated to our configuration by repeating the arguments for the O3 plane). The system is located at a point in the 45 directions. The object which couples magnetically to the D6 brane is the RR 1-form of Type IIA. Correspondingly $c$ measures the number of half D6 branes, mod 2, trapped inside the $\mathbf{R P}^{\mathbf{1}}$.

Let us summarize the situation after further applying $\mathrm{T}$ duality to other directions. An $O p$ plane along $012, \ldots, p-1$ and 6 , a half NS brane along 012345 and a half $\mathrm{D}(\mathrm{p}+2)$ brane along $012, \ldots, p-1,789$. When crossing a half NS brane the Op plane changes its $b$ charge and when crossing a half $D(p+2)$ brane the $O p$ plane changes its $c$ charge which is measured by a 'Wilson loop integral' of the RR $(5-p)$ form potential. This configuration exists for any $p$ which is 5 or less. $b$ measures the number of half NS branes in an $\mathbf{R P}^{\mathbf{2}}$ enclosing the configuration while $c$ measures the number of half $D(p+2)$ branes inside an $\mathbf{R P}^{\mathbf{5}-\mathbf{p}}$ which surrounds the configuration.

\subsection{T Duality}

Let us apply T-duality in a direction along the orientifold plane. An $O(p+1)$ plane wrapping a circle of radius $L$ turns after $\mathrm{T}$ duality to a pair of $O p$ planes on a circle of radius $L^{\prime}=1 / L$. Since we have (at least) 4 possible types for each $O p$ plane we expect 16 possible types for the wrapped $O(p+1)$ plane. 
Although this may sound surprising at first, we will demonstrate the 16 possibilities by analyzing the possible discrete torsions. The transverse space is $\mathbf{R P}^{\mathbf{7}-\mathbf{p}}$ with cohomologies given in appendix A, and we should find the 9 dimensional fields that can have discrete torsions. Before wrapping the $O(p+1)$ plane the fields with $\mathbf{Z}_{2}$ discrete torsions are $B_{N S}$ and $C_{5-(p+1)=4-p}$. After compactifying on $L$ there are two more such fields - the reduction of the metric on $L$ (an untwisted 1 form), and the reduction of $C_{6-p}$ on $L$. To find the relation with the $\mathrm{T}$ dual picture it is useful to list the various discrete torsions in both pictures

\begin{tabular}{|c||c|c|c|c|}
\hline$O(p+1)$ & $B_{N S}$ & $C_{4-p}$ & $g_{\mu \nu} / L$ & $C_{6-p} / L$ \\
\hline$O(p)$ & $B_{N S}$ & $C_{5-p} / L^{\prime}$ & $B_{N S} / L^{\prime}$ & $C_{5-p}$ \\
\hline
\end{tabular}

where the notation $C_{q} / L$ means "the form $C_{q}$ reduced on the circle $L$ ". Let us restate this mapping in terms of brane intersections

\begin{tabular}{|c||c|c|c|c|}
\hline$O(p+1)$ & NS5 & $D(p+3)$ & KK monopole & $\hat{D}(p+1)$ \\
\hline$O(p)$ & NS5 & $\hat{D}(p+2)$ & $\widehat{N S} 5$ & $D(p+2)$ \\
\hline
\end{tabular}

where the hat above a brane means that it does not wrap the circle.

Let us discuss some examples. An NS5 intersecting the $O(p+1)$ turns into an NS5 which intersects both of the $O p$ 's while an intersection with a $D(p+3)$ turns into a $D(p+2)$ which intersects only one out of the pair. If we want to get an RR discrete torsion on both $O p$ 's then we should intersect the $O(p+1)$ with a $D(p+1)$, one which does not wrap the circle (and still has 4 mixed directions relative to the $\mathrm{O}$ plane). If we take an $O p^{-}, O p^{+}$pair, which has zero charge, then it transforms into an $O(p+1)$ intersecting a KK monopole on the circle $L^{\prime}$ and must have zero charge as well.

\section{Review of M-lifts of Orientifolds}

In this section we review known lifts of orientifold planes to M theory. Such a lift requires lifting the $\mathbf{Z}_{2}$ action to $\mathrm{M}$ theory. The objects we describe are lifts of both orientifolds and orbifolds and accordingly are denoted as $O M p$ planes. Since the worldsheet formulation is lost in the lift, orientation reversal is meaningless. Nevertheless we shall sometimes continue to call them "orientifolds".

Lacking a fundamental definition of $\mathrm{M}$ theory, we are satisfied by specifying the $\mathbf{Z}_{2}$ action on the $11 \mathrm{~d}$ supergravity fields. An $O M p$ plane includes a transverse spatial reflection, so we look at $\mathrm{M}$ theory on $\mathbf{R}^{p, 1} \times \mathbf{R}^{10-p} / \mathbf{Z}_{2}$ where the first factor is the worldvolume of the $O M p$ plane, the $\mathbf{Z}_{2}$ in the second part is the reflection, and often it will be more convenient to replace the last factor with $\mathbf{T}^{10-p} / \mathbf{Z}_{2}$. The reflection 
determines the action on the metric $(g \rightarrow g)$. The action on the 3 -form $C$ is determined by requiring invariance of the topological term in the action $\int C \wedge G \wedge G$, where $G=d C$, to be

$$
C \rightarrow(-)^{p} C
$$

Supersymmetry provides another constraint. When acting on fermions, the inversion of $10-p$ coordinates squares to the identity for $10-p=0,1 \bmod 4$ and to $(-)^{F}$ for $10-p=2,3 \bmod 4$ (this is a consequence of $\left.\left(\Gamma_{1} \Gamma_{2} \ldots \Gamma_{n}\right)^{2}=(-)^{n(n-1) / 2}\right)$. Thus in order to have a supersymmetric orbifold and a $\mathbf{Z}_{2}$ action rather than a $\mathbf{Z}_{4}$ action we require

$$
p=1,2 \bmod 4 .
$$

We see that the $\mathbf{Z}_{2}$ objects intrinsic to $\mathrm{M}$ theory, are the OM1, OM2, OM5, OM6 and OM9. It is no surprise that the orientifolds intrinsic to $M$ theory include a 2 plane and a 5 plane which we denote by OM2 and OM5. We shall review the definition of these orientifolds and the way they were used to find the M lift of the O4 and the O2. Then we look for intersections of branes and orientifolds in $M$ theory, where it turns out that there is only one such configuration: a 1/2 M5 stuck on an OM2. After that we review the lift of another Type IIA orientifold, the O0, through an M theory object which we may call OM1.

We summarize here the results. The OM5 and OM2 carry charges given by

\begin{tabular}{|c|c|c|}
\hline$O M 2^{-}$ & $O M 2^{+}$ & OM5 \\
\hline$-1 / 16$ & $+3 / 16$ & $-1 / 2$ \\
\hline
\end{tabular}

where the $O \mathrm{M2}^{-}, \mathrm{OM}^{+}$are two discrete torsion variants of the $O M 2$ plane (which will be discussed later in subsection 3.2.1), and the charge is in units of bulk OM2 and OM5 branes. The other planes support a "twisted sector" matter rather than charge (they are neutral in 11d)

\begin{tabular}{|c|c|c|}
\hline$O M 1$ & $O M 6$ & OM9 \\
\hline a chiral fermion & a $7 \mathrm{~d} S U(2)$ vector multiplet & a 10d $E(8)$ vector multiplet \\
\hline
\end{tabular}

The OM6 and OM9 will not be discussed any further in this paper. The OM6 is M theory on $\mathbf{T}^{\mathbf{4}} / \mathbf{Z}_{\mathbf{2}}$ and the OM9 is a Hořava - Witten plane [41].

\subsection{The OM5 plane}

The "orientifold" $M$ on $\mathbf{T}^{5} / \mathbf{Z}_{2}$ was studied in [15, 19]. The "untwisted" sector has a $6 \mathrm{~d}$ gravitational anomaly that can be canceled by 16 tensor multiplets. Moreover, local anomaly cancellation would seem to require adding a twisted sector of $1 / 2$ a tensor 
multiplet at each of the 32 fixed points. This problem is avoided by using a different method of canceling the anomaly - assigning to each one of the fixed points a charge

$$
Q(O M 5)=-1 / 2
$$

in units of physical M5 branes. Note that while we can usually put a half brane on top of an orientifold plane, one cannot put half an M5 on an OM5 due to M theory flux quantization 42 .

\subsubsection{M-lift of the $\mathrm{O} 4$}

The OM5 can account for the O4 planes of Type IIA [20, 21] (see also [43]). When lifting the $\mathbf{Z}_{2}$ action from Type IIA to $\mathrm{M}$ theory it is required to specify the action on the circle $R_{11}$. Topologically there are three possible actions: the identity, a reversal and a shift through half a circle. The identity is interpreted as an OM5 wrapped on $R_{11}$. Reversal is not allowed since it would accumulate to an inversion in 6 directions which would break supersymmetry (3.2). A shift is the same as a non-trivial discrete torsion for the Type IIA 1-form $A$. It is exactly the discrete torsion which is present in the $\widetilde{O 4}^{ \pm}$planes. So "untilded" $(c=0)$ orientifolds M-lift to $\mathbf{R}^{5} / \mathbf{Z}_{2}$ while the "tilded" ones $(c=1)$ M-lift to $\left(\mathbf{R}^{5} \times \mathbf{S}^{\mathbf{1}}\right) / \mathbf{Z}_{\mathbf{2}}$ with the $\mathbf{Z}_{2}$ acting on the circle by a shift.

Let us look more at the shift orbifold $\left(\mathbf{R}^{5} \times \mathbf{S}^{\mathbf{1}}\right) / \mathbf{Z}_{\mathbf{2}}$. It has two kinds of minimal 1-cycles, one of them wraps $\mathbf{S}^{\mathbf{1}}$ ("the circle") and the other is a straight line between points identified by the $\mathbf{Z}_{2}$ action ("antipodal line"). For points away from the origin of $\mathbf{R}^{5}$ the circle is smaller, but as we approach the origin the antipodal line becomes smaller, and at the origin itself it is half the size of the circle.

We summarize the different $\mathrm{O} 4$ planes and their M theory origin

1. $O 4^{-}$. Charge $Q=-1 / 2$. This is simply an OM5 wrapping $R_{11}$.

2. $\widetilde{O_{4}}-$. Charge $Q=0$. This is the smooth shift orbifold, and as such indeed does not carry charge. (Recall that a bound state of an OM5 and a half M5 is not allowed).

3. $O 4^{+}$. $Q=+1 / 2$. This is an OM5 with a full stuck M5. (It is stuck by imposing as Wilson loop an element of $O(2)$ which is not connected to the identity [21]).

4. $\widetilde{\mathrm{O4}^{+}}$. Charge $Q=+1 / 2$. This is the shift orbifold with a stuck M5 at the origin on the circle of half radius.

The two types of $\widetilde{O 4}$ planes were conjectured to be related to the two elements of $\pi_{4}(S p(n))=\mathbf{Z}_{2}$. 


\subsection{The OM2 plane}

The OM2 orientifold was studied in [17] (see also [44). The charge can be found by considering the interaction $-\int C \wedge I_{8}(R)$ for $\mathrm{M}$ theory on $X=\mathbf{T}^{\mathbf{8}} / \mathbf{Z}_{\mathbf{2}}$. The effective charge is $-\int_{X} I_{8}(R)=-\chi / 24$. Although this space is singular, we can define its "resolved cohomologies" by adding to the invariant (untwisted) cohomologies an extra 256 cohomologies (in $H^{2,2}$ ) from the RR twisted sector. This totals the Euler characteristic to 384 and so the total charge to -16 . Dividing by 256 , the number of fixed points, we find $Q=-1 / 16$ in units of M2 charge.

This orientifold allows a variant due to a discrete flux. The transverse space is $\mathbf{R P}^{7}$ and the only field strength form in $\mathrm{M}$ theory is the 4-form $G$, so we are interested in the cohomology $H^{4}\left(\mathbf{R P}^{7}, \mathbf{Z}\right)=\mathbf{Z}_{2}$ (Appendix A). The discrete torsion adds a charge

of a $+1 / 4=-\frac{1}{2} \int_{\mathbf{R P}^{7}} \frac{C}{2 \pi} \wedge \frac{G}{2 \pi}$ in units of M2. We denote the OM2 with trivial discrete torsion by $O M 2^{-}$and the one with non-trivial torsion by $\mathrm{OM2}^{+}$. Their charges are summarized by

$$
\begin{array}{r}
Q\left(O M 2^{-}\right)=-1 / 16 \\
Q\left(O M 2^{+}\right)=Q\left(O M 2^{-}\right)+1 / 4=+3 / 16 .
\end{array}
$$

\subsubsection{The brane - orientifold intersection in $M$ theory}

The $O \mathrm{M2}^{+}$can be realized by branes. To do that we put a half M5 brane on an $O M 2^{-}$plane. This is an analogue (the only one) of the Type II configurations in section (2).

Note that like the case of the OM5, one cannot attach a half M2 brane on top of an OM2 due to M theory flux quantization 42.

Once we compactify $\mathrm{M}$ theory we get (at least) two more possibilities for brane intersections. One possible configuration comes from lifting OF1 and D6 with charge jump of 1/16. It lifts to a wound OM2 intersecting a KK6 - a Kaluza Klein monopole (section 5.1). Another configuration is the lift of an OF1 intersecting a D2, that is a wound OM2 intersecting with a transverse M2 with a tension jump of +1 (sections 4.3, 5.1). An inspection of this example shows that the jump of one unit of F1 charge is actually represented by a physical wound M2 brane which is stretched, with its mirror, like a $\mathrm{T}$ shaped brane (this is the M-lift of a double F1 ending on a D2 [45]).

\subsubsection{M-lift of the $\mathrm{O} 2$}

Once one identifies the $\mathrm{M}$ theory objects, the $O M 2^{-}$and the $O M 2^{+}$, one can go on and find the M-lift of the O2 planes in Type IIA [17, 18]. Actually, originally it must have been easier to go in the opposite direction and determine the charges of the OM planes from the $\mathrm{O} 2$ planes. Like the case of M-lifting the O4, we need to lift the $\mathbf{Z}_{2}$ 
action to $R_{11}$. This time it must be a reversal - it cannot be the identity because of the susy constraint on $p(3.2)$. So in $\mathrm{M}$ theory there are actually two fixed planes located at the two fixed points on the circle.

The different $\mathrm{O} 2$ planes and their $\mathrm{M}$ theory origin are described by

1. $O 2^{-}$. Charge $Q=-1 / 8$. This is a pair of $O M 2^{-}$planes: $-1 / 8=2 \times-1 / 16$.

2. $\widetilde{O 2^{-}}$. Charge $Q=-1 / 8+1 / 2=+3 / 8$. This is a pair of $O M 2^{+}$planes: $-3 / 8=2 \times-3 / 16$.

3. $\mathrm{O2}^{+}, \widetilde{\mathrm{O2}^{+}}$. Both have $Q=+1 / 8$. This is a composite pair of an $O M 2^{-}$with an $\mathrm{OM2}^{+}, 1 / 8=-1 / 16+3 / 16$. The two possible O2's correspond to the possible ordering of the OM2's. This can be seen by intersecting an $\mathrm{O}^{+}$with a $\mathrm{D} 4$ brane. After lifting to $\mathrm{M}$ theory and using the intersection rule explained in section (2), one finds that the $\widetilde{\mathrm{O2}^{+}}$has the reversed order of OM2's.

\subsection{The OM1 line}

Let us consider the M-lift of the orientifold point, the O0, of Type IIA. We take the action on $R_{11}$ to be the identity (a reversal is not allowed by equation 3.2), so we consider $\mathrm{M}$ theory on $R_{11} \times \mathbf{S}^{\mathbf{1}} \times \mathbf{R}^{\mathbf{9}} / \mathbf{Z}_{\mathbf{2}}$. A computation of $2 \mathrm{~d}$ Gravitational anomalies for $M$ theory on $\mathbf{T}^{\mathbf{9}} / \mathbf{Z}_{\mathbf{2}}$ suggests that there is a chiral fermion on every fixed line as an "M theory twisted sector" [15, 46]. An independent evidence for the existence of a chiral fermion on the fixed line comes from the computation of the Witten index of $S p(N), S O(N)$ matrix quantum mechanics [47, 16]. We may call this line an OM1 orientifold.

It is interesting to get the "twisted sector" which is described above truly from a twisted sector of string theory (see also [15, 48]). In order to get a $2 \mathrm{~d}$ model one needs to compactify on an 8 manifold, and Type IIB on $\mathbf{T}^{\mathbf{8}} / \mathbf{Z}_{\mathbf{2}}$ has the right action on the fields (this is the orbifold which we will call $O P 1_{B}$ in section 5.1). Type IIA divided by $I_{8} \cdot(-)^{F_{L}}$ would also do, but we will stay with the more geometric example. The $O P 1_{B}$ has a twisted sector from the 4 form wrapping the 256 resolved $H^{2,2}$ cohomologies (49] describes the computation of twisted sectors in general). These scalars are chiral as they inherit their self duality from the 4 form. Thus we get one chiral scalar for each of the 256 fixed lines. Comparing with the 512 chiral fermions of $\mathrm{M}$ theory on $\mathbf{T}^{\mathbf{9}} / \mathbf{Z}_{\mathbf{2}}$ we see that they could match by bosonization provided the periodicity of the scalars is at the free fermionic value, as it should. This actually gives a nice realization of 2 dimensional bosonization as implied by a lift to $\mathrm{M}$ theory.

As we consider here the OM1 on the $R_{11}$ circle there are two possible boundary

conditions for the fermion. The Neveu-Schwarz boundary conditions correspond to an 
O0 with trivial $R R$ discrete torsion $\left(c_{R R}=0\right)$, and Ramond corresponds to $c_{R R}=1$. This is verified by a computation of the Casimir energy in the two cases, which matches the $\mathrm{O} 0$ mass

$$
M(O 0)= \pm 1 / 32
$$

where the units are of momentum quanta along the circle.

Let us summarize the different $\mathrm{O} 0$ planes and their $\mathrm{M}$ theory origin

1. $O 0^{-}$. Charge $Q=-1 / 32$. This is an OM1 with NS boundary conditions, and with integral momentum.

2. $\widetilde{O 0^{-}}$. Charge $Q=-1 / 32+1 / 2$. This OM1 has NS boundary conditions, but carries half-integer momentum.

3. $\mathrm{O0}^{+}, \widetilde{\mathrm{O0}^{+}}$. Both have $Q=+1 / 32$. These OM1's are in the Ramond sector, and there are two of them due to the zero mode which generates a degenerate ground state.

\section{M lifts of Type IIB Orientifolds}

In this section we describe (new) M-lifts of various orientifold planes in Type IIB, while paying special attention to the transformation properties under $S L(2, \mathbf{Z})$.

We start by M-lifting the O3 plane, where we get a nice geometric/ microscopic realization of the $S L(2, \mathbf{Z})$ symmetry. Then we discuss the O1 and O5 planes. Their S duals, which we call OF1 and ON5, are constructed, and will be further discussed in section 5. The ON5 was already discussed in some works (see [36 for a recent review on this plane), and the OF1 was discussed in [50].

The method is to recall the $\mathrm{M}$ theory origin of a $\mathrm{Dp}$ brane in terms of $\mathrm{M}$ branes and then to find the analogous construction of an Op plane in terms of the OM planes which were reviewed in the previous section. The basic correspondence is between $\mathrm{M}$ theory on $\mathbf{T}_{\mathbf{M}}^{\mathbf{2}}$ and IIB on a circle of radius $L_{I I B}$, so we should always distinguish two cases according to whether the Dp brane wraps $L_{I I B}$ or not. At weak coupling the

$\mathbf{T}_{M}^{2}$ has a short side and a long side, such that their ratio is the string coupling (when the $R R$ axion vanishes).

\section{$4.1 \mathrm{O} 3$}

Let us recall the M-lift of the D3 brane. A D3 which does not wrap $L_{I I B}$ is an M5 wrapping the torus, while a D3 which wraps $L_{I I B}$ is the M2. 


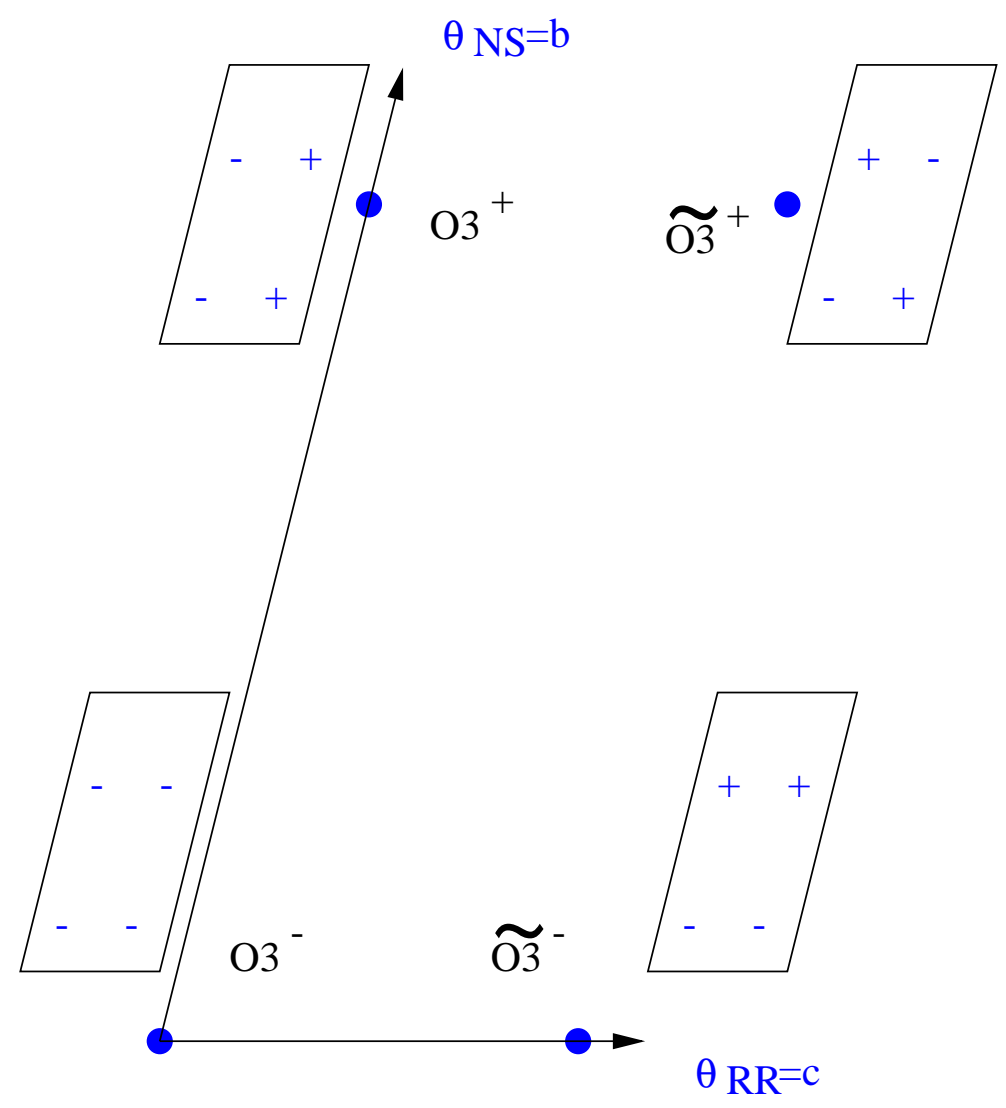

Figure 3: The four types of $O 3$ orientifolds lifted to $M$ theory. The \pm signs stand for $O M 2^{ \pm}$ planes inside the $\mathrm{M}$ theory torus.

The four kinds of O3 planes have the following charges (in D3 units)

$$
\begin{array}{r}
Q\left(\widetilde{O 3^{-}}\right)=-1 / 4, \\
Q\left(\widetilde{O 3^{-}}\right)=Q\left(O 3^{+}\right)=Q\left(\widetilde{O 3^{+}}\right)=+1 / 4 .
\end{array}
$$

We start by lifting an $\mathrm{O} 3$ which wraps $L_{I I B}$, for otherwise the circle $L_{I I B}$ is inverted as well and we get two $\mathrm{O} 3$ planes at the two fixed points. Since the M-lift of a D3 brane which wraps $L_{I I B}$ is an M2 which does not wrap $\mathbf{T}_{M}^{2}$, we should take an OM2 which does not wrap $\mathbf{T}_{M}^{2}$. Because of the compactness of $\mathbf{T}_{M}^{2}$ we are considering actually four OM2 planes. The simplest possibility is to take four $O M 2^{-}$planes. One checks that the total charge $4 \times(-1 / 16)=-1 / 4$ fits the $O 3^{-}$plane as expected.

To get the other $\mathrm{O} 3$ planes ${ }^{4}$ we use the brane intersection picture of section 2. For example, to get the M-lift of the $O 3^{+}$we should intersect the $O 3^{-}$with an NS5 brane. The lift of the NS5 is an M5 wrapping the long side. Using the basic intersection in

\footnotetext{
${ }^{4}$ A.H. would like to thank Jacques Distler for discussions on related points.
} 
$\mathrm{M}$ theory (section 3.2.1) we see that we end up with two $O M 2^{+}$planes along the long side, and two other $O M 2^{-}$planes, as in figure 3. The charges fit since $2 \times(-1 / 16)+$ $2 \times(+3 / 16)=+1 / 4$. A similar argument works for the $\widetilde{\mathrm{O3}^{-}}$and the $\widetilde{\mathrm{O3}^{+}}$planes by replacing the NS5 branes with a D5 brane or a $(1,1)$ brane respectively. Figure (3) summarizes the various configurations.

Recall that the $S L(2, \mathbf{Z})$ properties of O3 planes can be described in a diagram such as figure 3 [32, 33, where $S L(2, \mathbf{Z})$ acts on the torus in the diagram according the its natural action on $\left(\mathbf{Z}_{2}\right)^{2}$. This action is clearly visible from our M-lift into $\mathbf{T}_{M}^{2}$.

There is an alternative way of finding the $\mathrm{M}$ lift of an O3 which uses $\mathrm{T}$ duality (section 2.2). Under $\mathrm{T}$ duality an $\mathrm{O} 3$ that wraps $L_{I I B}$ turns into a pair of $\mathrm{O} 2$ planes, each one of which can be lifted to a pair of OM2 planes, as in section 3.2.2, giving 4 OM2 planes as above. In this way one can recover the different lifts for the different variants.

One may wonder about other choices for the signs of the four OM2 planes. The ones with an odd number of signs cannot be constructed by intersecting the $03^{-}$with a Type IIB 5-brane as the 5-brane must intersect exactly two OM2 planes (because the M5 is oriented). Nevertheless, such configurations can be constructed making use of the large but compact circle $L_{I I B}$, by intersecting the wound $\mathrm{O} 3$ (wound on $L_{I I B}$ ) with a KK monopole (on $L_{I I B}$ ) as in (2.6). One gets a configuration with three $O M 2^{-}$ and one $\mathrm{OM2}^{+}$and total charge zero. Intersecting now with an NS5 would give the other possibility - one $O M 2^{-}$and three $O M 2^{+}$. The case of $4 O M 2^{+}$planes is probably equivalent to four $\mathrm{OM}^{-}$planes with an additional M2 brane.

Now we turn to an O3 which does not wrap $L_{I I B}$. It is actually a pair of O3 planes, and we may T- Dualize them into an $\mathrm{O} 4$ plane as in section (2.2). The M-lift of the latter was described already in section 3.1.1 in terms of OM5 planes.

\subsection{O5 - ON5}

Let us discuss $\mathrm{O} 5$ planes which wrap $L_{I I B}$, both because we are less interested in a pair of O5's which we would have had if the compact direction were inverted, and since we are interested in configurations which lift to M5 branes rather than KK monopoles. ${ }^{5}$ For 5 branes, A $(p, q) 5$ brane which wraps $L_{I I B}$ lifts to an M5 wrapping a $(p, q)$ cycle of $\mathbf{T}_{M}^{2}$. By analogy, we attempt to lift the O5 to an OM5 wrapping the short side of $\mathbf{T}_{M}^{2}$. Such an OM5 plane is actually a pair of OM5's because of the transverse compact coordinate on $\mathbf{T}_{M}^{2}$. We check that the charges match: $2 \times Q(O M 5)=2 \times(-1 / 2)=-1=Q(O 5)$.

\footnotetext{
${ }^{5} \mathrm{An}$ alternative $\mathrm{M}$ lift of the $\mathrm{O} 5$, the $O N 5_{B}$ and their variants was given in [34], using OM6 planes and more elaborate quotients rather than OM5 planes.
} 
So far we have discussed the $\mathrm{O}^{-}$. We would like to construct other discrete torsion variants of the $\mathrm{O} 5$, ones which are independent of the compactification on $L_{I I B}$, namely, those which are not related to forms that were reduced on $L_{I I B}$. This can be done by turning on discrete torsions for $\mathrm{M}$ theory on the torus.

Performing $S L(2, \mathbf{Z})$ we can get a family of $(p, q)$ O5 planes, such that the charge of a $(p, q) O 5^{-}$plane is -1 in units of a $(p, q)$ 5-brane. In particular we can consider a $(0,1)$ O5 plane which we call $O N 5_{B}$ because it is charged under the same field that couples to the NS5 brane of Type IIB (the charge is -1 in units of the NS5 charge). The system of $O N 5_{B}^{-}$together with an NS5 brane can be identified to be the $I I B / I_{4}(-)^{F_{L}}$ orbifold and can be called $O N 5_{B}^{0}$ (section 5.2; see [36] for a more detailed discussion). A set of N NS5 branes in the vicinity of an $O N 5_{B}$ results in $6 \mathrm{~d}$ worldvolume gauge theory, the same as a set of D5 branes near an 05 plane, with the gauge group being one of $S O(2 N), S O(2 N+1), S p(N)$ according to the type of the $O N 5_{B}$.

Since NS5 branes exist both in Type IIA and in Type IIB, one might expect the ON5 to exist in Type IIA as well. Indeed, the $I I A / I_{4}(-)^{F_{L}}$ orbifold (section 5.2), which we call an $O N 5_{A}^{0}$, is a system composed of an $O N 5_{A}$ with an NS5 brane. The $O N 5_{A}^{-}$ has the property that when N NS5 branes coincide with it, the worldvolume theory is a $(2,0)$ CFT with a global symmetry group $S O(2 N)$, and this is the only possible variant.

\section{$4.3 \mathrm{O} 1$ - OF1}

The case of $\mathrm{O} 1$ is quite similar to the O5. Consider an O1 which does not wrap $L_{I I B}$ (so there is actually a pair of O1's). As a D1 which does not wrap $L_{I I B}$ M-lifts to a membrane which wrap the long side of the torus, we should try an OM2 plane wrapping the long side (so again there are actually two of them because of the transverse short side). Let us check the charges: $2 \times(-1 / 16)$ for the pair of O1's, indeed equals $2 \times(-1 / 16)$ for the pair of OM2's.

For O1 planes which wrap $L_{I I B}$, we recall that a D1 wrapping $L_{I I B}$ is described by a unit of momentum along the short side of $\mathbf{T}_{M}^{2}$. So we try to wrap an OM1 along the short side of $\mathbf{T}_{M}^{2}$ as its mass scales like units of momentum (actually it is a pair of OM1's due to the transverse long side). The charges work out for an $O 1^{-}$being made of a pair of $O M 1^{-}:-1 / 16=2 \times(-1 / 32)$.

One can get other variants of the $\mathrm{O} 1$ by lifting brane intersection to $\mathrm{M}$ theory. A new configuration happens for a pair of $01^{-}$'s which do not wrap $L_{I I B}$ and are intersected by a D3 which does. After the intersection we get a pair of $\widetilde{O^{-}}$, and so the tension jump is $2 \times+1 / 2=+1$. By lifting to $\mathrm{M}$ theory we learn that the intersection of a wound OM2 with a transverse M2 gives a +1 tension jump. 
Performing $S L(2, \mathbf{Z})$ we can get a family of $(p, q)$ O1 planes, such that the charge of a $(p, q) O 1^{-}$plane is the same as for a $(p, q)$ string. In particular we can consider a $(1,0)$ O1 plane which we call $O F 1_{B}$ because it is charged under the same field that couples to the fundamental string. We will see that the $O F 1_{B}$ can be identified with the $I I B / I_{8}(-)^{F_{L}}$ orbifold (section 5.1). Since fundamental strings exist both in IIA and in IIB, one might expect the OF1 to exist in Type IIA as well. Indeed, this is the $I I A /(-)^{F_{L}}$ orbifold (section 5.1), and we call it an $O F 1_{A}$. The perturbative orbifold variants may be referred to as $O F 1_{B}^{0}, O F 1_{A}^{0}$.

\section{Orbifolds, Orientifolds and New Variants}

Here we discuss the relations between orientifolds and orbifolds and their variants. We start with lines, then 5 planes and then the O0. Throughout this section, when we identify a perturbative orbifold with some plane which is a dual of an orientifold, it should be borne in mind that the identification holds only for one variant of the plane, possibly with some extra matter, and all other variants are produced by changing non-perturbative discrete torsions.

\subsection{Orbifold lines}

Orbifold lines together with the O1 form a family connected by dualities. Table 1 is our roadmap for these connections. We will first explore this map and then present some results on the tensions of discrete torsion variants and a relation with $\mathrm{K}$ theory.

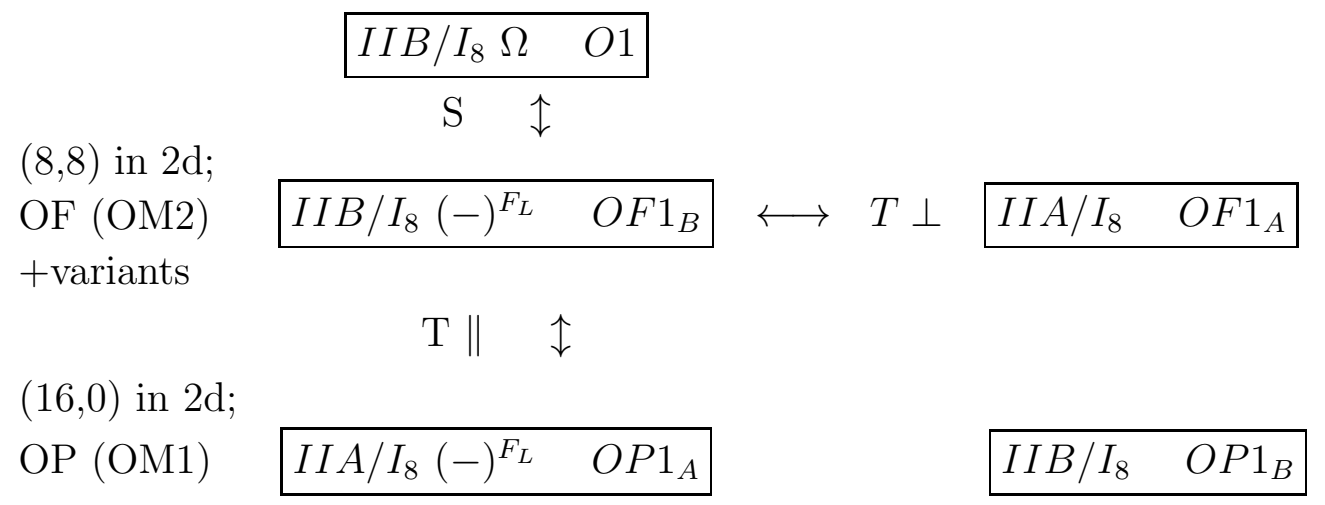

Table 1: Orbifold and orientifold lines. In this table the charges and fluxes of the various $O$ planes are not specified and may change, depending on the particular case.

Our starting point is the $\mathrm{O} 1$, that is, $I I B / I_{8} \Omega$. It carries $\mathrm{D} 1$ charge and the forms $B_{N S}, C_{0}, C_{4}$ are odd (twisted) under it. It has discrete torsion variants due to $H_{N S}, * H_{N S}, G_{5}, G_{1}$ or in terms of brane intersections due to the NS5, F1, D3 and a 7 
brane. The 7 brane must allow a D1 charged object to end on it, and so it should be a $(0,1) 7$ brane rather than a $(1,0)$ D7.

We denote the $\mathrm{S}$ dual of the $\mathrm{O} 1$ by $O F 1_{B}$ as $\mathrm{S}$ duality replaces a $\mathrm{D} 1$ charge with an F1 charge. $\mathrm{S}$ duality replaces $\Omega$ with $(-)^{F_{L}}$ and so this orbifold is $I I B / I_{8}(-)^{F_{L} 6}$. The odd (twisted) forms under the projection are $C_{2}, C_{0}, C_{4}, C_{6}$ and we get 16 (!) discrete torsion variants from all four, or in terms of branes from intersections with D1,D3,D5,D7. Since an F string can end on any D brane, it is natural that each intersection with a $\mathrm{D}$ brane is allowed and gives a new variant.

Operating on the $O F 1_{B}$ with a $\mathrm{T}$ duality in a direction transverse to the fixed line gives an orbifold which we denote by $O F 1_{A}$, just as this operation acting on the $\mathrm{F}$ string of IIB would give the F string of Type IIA. Such a $\mathrm{T}$ duality is accompanied by an additional $(-)^{F_{L}}$, so operating on $I I B / I_{8}(-)^{F_{L}} \equiv O F 1_{B}$ we get the $I I A / I_{8}$ orbifold. This orbifold has 8 variants due to intersections with $\mathrm{D}$ branes $D 2, D 4, D 6$ or their respective forms $G_{2}, G_{4}, G_{6}$. A D8 intersection is different because the associated $G_{0}$ form has $H^{0}\left(\mathbf{R P}^{\mathbf{7}}\right)=\mathbf{Z}$ cohomology rather than $\mathbf{Z}_{2}$ and is interpreted as a change in the Type IIA cosmological constant. The D0 is not in the list since it has no cohomology $H^{8}\left(\mathbf{R P}^{\mathbf{7}}\right)=\mathbf{0}$ (but it may produce variants nevertheless).

Other orbifolds can be constructed now by compactifying an OF1 line on a circle and performing parallel T duality. This time one does not add an extra $(-)^{F_{L}}$. We get $I I B / I_{8}$ and $I I A / I_{8}(-)^{F_{L}}$. These orbifolds do not have discrete torsion variants (when uncompactified). By $\mathrm{T}$ duality they carry a momentum charge, so we denote them by $O P 1_{A}, O P 1_{B}$.

The $\mathrm{M}$ lift of the O-lines can be found by looking at their charges. After recalling the $\mathrm{M}$ lift of the $\mathrm{F}$ string we conclude that the OF1 planes must be wrapping modes of the OM2. The OP1 planes, on the other hand, are an unwrapped OM1. Since the OM1 carries a chiral fermion (section 3.3) after being compactified its Casimir energy will give the required momentum charge.

Let us now find the tensions of some of the discrete torsion variants of the OF1 lines (table 2). The tension of a bare OF1 is $-1 / 16$ (in F string units) by $\mathrm{S}$ duality with the $\mathrm{O} 1$. It is consistent with the $\mathrm{M}$ description as an OM2 wrapping the 11'th dimension, since the OM2 has tension -1/16 (in M2 units).

- To compute the tension of an $O F 1_{B}$ after intersecting a D5, consider performing $\mathrm{S}$ duality to an $\mathrm{O} 1$ which upon intersecting an NS5 changes from $\mathrm{O1}^{-}$of tension $-1 / 16$ to an $O 1^{+}$of tension $+1 / 16$ (so the jump is $+1 / 8$ ).

\footnotetext{
${ }^{6}$ To be more precise, one should note that the $\mathrm{O}^{-}{ }^{-}$, which is the $\mathrm{O} 1$ plane with no discrete fluxes carries charge $-\frac{1}{16}$, which must be cancelled by adding non-perturbative discrete torsion and/ or extra matter, since the orbifold Type $I I B$ on $\mathbf{T}^{\mathbf{8}} / \mathbf{Z}_{\mathbf{2}}$ has 0 charge.
} 


\begin{tabular}{|c|c|c|c|c|c|c|}
\hline D1 & D2 & D3 & D4 & D5 & D6 & D7 \\
\hline$+2 ?$ & +1 & $+1 / 2$ & $+1 / 4$ & $+1 / 8$ & $+1 / 16 ?$ & $+1 / 32 ?$ \\
\hline
\end{tabular}

Table 2: Tension jumps of the OF1 after various intersections. Question marks denote charges for which more consistency checks are needed.

- The intersection of an $O F 1_{A}$ with a $\mathrm{D} 4$ can be $\mathrm{M}$ lifted to the basic intersection of an OM2 with an M5 (section 3.2.1), and so the tension jump is $+1 / 4$.

- The tension jump of an $O F 1_{B}$ intersecting with a D3 is found again by $\mathrm{S}$ duality to be $+1 / 2$.

- The tension jump of an $O F 1_{A}$ intersecting a D2 is +1 . This is a consequence of the $\mathrm{M}$ theory configuration found in section 4.3 , where a wound OM2 intersects a transverse M2. Since the tension jump is integral there is nothing to prevent a whole F1 to separate from this variant.

- We seem to get a rule that after intersecting a Dp brane the jump is $2^{2-p}$, which is consistent with T duality. For the D6 this rule has an independent check. A D6 intersection corresponds after an $\mathrm{M}$ lift to a shift in $R_{11}$. So this $O F 1_{A}$ variant lifts to $\mathrm{M}$ theory on the smooth manifold $\left(\mathbf{T}^{\mathbf{8}} \times \mathbf{S}^{\mathbf{1}}\right) / \mathbf{Z}_{\mathbf{2}}$ where the $\mathbf{Z}_{2}$ acts by inversion on the first factor and by a shift on the second. As a smooth manifold it carries zero tension, in agreement with a $-1 / 16$ jump.

- The intersection of an $O F 1_{A}$ with a $\mathrm{D} 0$ does not have a discrete cohomology as $H^{8}\left(\mathbf{R P}^{\mathbf{7}}\right)=\mathbf{0}$ so it is not clear whether it gives a new variant. If there is a new variant corresponding to intersection of the OF1 with D0 the jump in its charge is 4 . Conservation of the fundamental string charge then implies that 4 physical fundamental strings must enter the D0 together with the $O F 1^{-}$plane. This is indeed consistent with the "fork" configuration of [40] which gives some support for its existence.

One can discuss OF1 orbifolds with a discrete torsion from several forms turned on at the same time. It would be interesting to find their tensions.

The above variants have an interesting relation with $\mathrm{K}$ theory. It is simpler to consider $O F 1_{A}=I I A / I_{8}$. Discrete torsion variants are classified by the reduced cohomology $H^{*}\left(\mathbf{R P}^{\mathbf{7}}\right)=\mathbf{Z}_{\mathbf{2}} \oplus \mathbf{Z}_{\mathbf{2}} \oplus \mathbf{Z}_{\mathbf{2}}$. However, it was recently claimed that the correct classifying group is the reduced $\mathrm{K}$ group 24 $K\left(\mathbf{R P}^{\mathbf{7}}\right)=\mathbf{Z}_{\mathbf{8}}$ ("reduced" simply means in both cases that we do not write down a trivial $\mathbf{Z}$ factor). The $\mathrm{K}$ group 
is actually a ring which differs from the standard $\mathbf{Z}_{8}$ and is defined by the following relations on its generator $x$

$$
\begin{array}{r}
8 x=0 \\
x^{2}=-2 x
\end{array}
$$

Note that both the cohomology and the K ring have the same order (8) whereas their structures differ. It would be interesting to elucidate the role of the algebraic structure.

\subsection{Orbifold 5 planes}

We can take a similar tour of orbifold 5 planes, with table 3 as our road map.

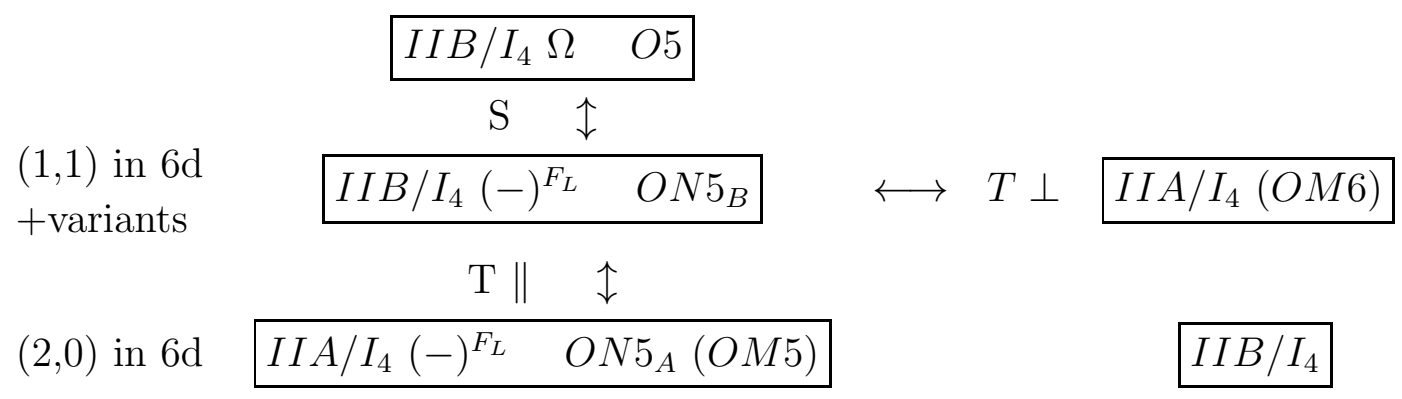

Table 3: Orbifold and orientifold 5 planes

We start with the $\mathrm{O} 5$ orientifold. It has charge -1 in units of D5, and it has a pair of discrete torsions due to the forms $G_{1}, H_{N S}$, or in terms of branes due to intersection with $D 7, N S 5$.

$\mathrm{S}$ duality creates the orbifold $I I B / I_{4}(-)^{F_{L}}$, which we denote by $O N 5_{B}$ since it carries NS5 charge. In order to cancel the charge one needs to add to the $O N_{5}$ plane an NS5 and this is actually the perturbative orbifold. The matter living on the NS5 of Type IIB is, of course, in $(1,1) 6 \mathrm{~d}$ multiplets. It has variants from the RR forms $G_{1}, G_{3}$, allowing all the $S O, S p$ gauge groups.

Performing $\mathrm{T}$ duality parallel to an $O N 5_{B}$ gives Type IIA $/ I_{4}(-)^{F_{L}}$, which we denote by $O N 5_{A}$, because it has NS5 charge as well. It carries matter in $(2,0) 6 \mathrm{~d}$ multiplets. If N NS5 branes are added to the orbifold we get a $(2,0)$ theory with $S O(2 N)$ group.

We can also perform $\mathrm{T}$ duality transverse to the orbifold planes. We get $I I A / I_{4}$ and $I I B / I_{4}$. The first orbifold has a variant due to $G_{2}$. These orbifolds are known to carry non perturbative matter - a $(1,1)$ theory in the first case, and $(2,0)$ in the second.

Let us consider the M lifts of Type IIA orbifold 5-planes. We can imagine two $\mathrm{M}$ theory orbifolds that would give us 5 planes in Type IIA, either an OM6 wound 
on $R_{11}$ or an unwound OM5. By comparing the action on the fields we find that the $O N 5_{A}=I I A / I_{4}(-)^{F_{L}}$ lifts to the unwound OM5 while $I I A / I_{4}$ lifts to a wound OM6. The case of Type IIB can be discussed as well, but it has more detail because one needs to specify whether the 5 plane wraps $L_{I I B}$ or not.

\subsection{New variants of the O0 plane}

For the low dimensional orientifolds, the $\mathrm{O} 0$ and the $\mathrm{O} 1$, a discrete torsion analysis predicts the existence of more than two $\mathbf{Z}_{2}$ parameters. For the $\mathrm{O} 1$ we saw that there are four $\mathbf{Z}_{2}$ parameters. In addition to the usual $(b, c)=H_{N S}, G_{5}$ there are also a pair of $\mathbf{Z}_{2}$ 's from $G_{1}$ and $* H_{N S}$.

Similarly, for the $\mathrm{O} 0$ we can analyze the possible discrete torsions. In addition to the expected pair of $\mathbf{Z}_{2}$ parameters $(b, c)=H_{N S}, G_{6}$, there is a third $\mathbf{Z}_{2}$ from $G_{2}$. After an $\mathrm{M}$ lift, this additional discrete torsion is nothing but a shift in $R_{11}$, the possibility which was not considered section 3.3. It would be interesting to find the mass of the 4 variants with $\left[G_{2}\right] \neq 0$.

\section{Miscellaneous applications}

\subsection{The spectrum of $4 \mathbf{d} \mathcal{N}=4$ with $S O, S p$ gauge group}

We know that every orientifold 3-plane gives rise to a 4 dimensional gauge theory on D3 probes parallel to it. Each O3 plane gives rise to a theory with a different gauge group $G$, according to

1. For $O 3^{-} G=S O(2 N)$.

2. For $\widetilde{O 3^{-}} G=S O(2 N+1)$.

3. For both $O 3^{+}, \widetilde{O 3^{+}}$the gauge group is $G=S p(N)$.

Since both $\mathrm{O3}^{+}$and $\widetilde{\mathrm{O3}^{+}}$have the same gauge group one may ask how do the two theories differ. It is clear that the theory with $\widetilde{O 3^{+}}$is an $S L(2, \mathbf{Z})$ transform of the one with $O 3^{+}$by the element

$$
T=\left[\begin{array}{ll}
1 & 1 \\
0 & 1
\end{array}\right] .
$$

We would like to show how this difference manifests itself in one of the basic measurables of the theory, the spectrum of $1 / 2$ BPS states.

By Definition we know that the $\mathrm{W}$ bosons lie in the root lattice of the gauge group $G$. Knowing the lattice of the $\mathrm{W}$ bosons and the $S L(2, \mathbf{Z})$ transformation which acts 
naturally both on the charges of the states and on the discrete charges of the orientifolds allows us to characterize the $1 / 2$ BPS spectrum as follows

\begin{tabular}{|r|c|c|c|c|}
\hline$(b, c)=$ & $\begin{array}{c}\text { O3 }^{-} \\
(0,0)\end{array}$ & $\begin{array}{c}\text { O3 }^{-} \\
(0,1)\end{array}$ & $\begin{array}{c}\text { O3 }^{+} \\
(1,0)\end{array}$ & $\begin{array}{c}\text { 33 }^{+} \\
(1,1)\end{array}$ \\
\hline \hline$(p, q)=(1,0) \bmod 2$ & $\mathrm{D}$ & $\mathrm{B}$ & $\mathrm{C}$ & $\mathrm{C}$ \\
$(p, q)=(0,1) \bmod 2$ & $\mathrm{D}$ & $\mathrm{C}$ & $\mathrm{B}$ & $\mathrm{C}$ \\
$(p, q)=(1,1) \bmod 2$ & $\mathrm{D}$ & $\mathrm{C}$ & $\mathrm{C}$ & $\mathrm{B}$ \\
\hline
\end{tabular}

Here $D$ denotes that the states lie in a $D=S O(2 N)$ lattice, $B$ is a $B=S O(2 N+1)$ lattice and $C$ is a $C=S p(N)$ lattice. We get that the charge lattice is of type B iff $(p, q)=(c, b) \bmod 2$.

Note that the difference between $\mathrm{O3}^{+}$and $\widetilde{\mathrm{O3}^{+}}$is manifest in their spectrum of monopoles and dyons - whereas the monopoles of the $O 3^{+}$theory lie in the $B$ lattice (the dual lattice), the monopoles of the $\widetilde{O 3^{+}}$theory lie in a $C$ lattice just like the W bosons.

\subsection{A comment on allowed BPS stated and $\mathrm{Z}_{2}$ charges}

Consider a configuration with an $O p$ plane and a physical Dp brane away from it. Our aim is to ask what are the allowed BPS configurations which can stretch in between the Dp brane and its image. For the simplest case, with $O p^{-}$the gauge group is $S O(2)$. It is known that a fundamental string stretched between the Dp brane and its image does not lead to a BPS state but rather, as Sen shows in detail in [51, to a non-BPS state. This happens because the BPS ground state is projected out, so the lowest state is the next massive level which is not BPS.

We would like to extend this discussion to monopole solutions and, when possible, to dyonic states. The first example is for $p=3$. Using the results of the previous subsection we find that the allowed BPS states are described by the following table

\begin{tabular}{|c|l|l|l|l|}
\hline$(\mathrm{b}, \mathrm{c})$ & $(0,0)$ & $(1,0)$ & $(0,1)$ & $(1,1)$ \\
\hline \hline $\begin{array}{c}\text { Fundamental String } \\
\text { D1 brane }\end{array}$ & no & yes & no & yes \\
no & yes & yes \\
\hline
\end{tabular}

It is easy to see from this table that a brane is allowed as a BPS state whenever the $\mathbf{Z}_{2}$ of the two form which couples to it electrically is non trivial.

This leads to the following generalization for any $p$. Consider a Dp brane and its image under a reflection by an $O p$ plane. Then BPS states arise when either a fundamental string or a Dp-2 brane is stretched between the brane and its image, 
according to the following table:

\begin{tabular}{|c|c|c|c|c|}
\hline$(\mathrm{b}, \mathrm{c})$ & $(0,0)$ & $(1,0)$ & $(0,1)$ & $(1,1)$ \\
\hline \hline Fundamental String & no & yes & no & yes \\
Dp-2 brane & no & no & yes & yes \\
\hline
\end{tabular}

Unlike the previous case, the simple rule that a brane is allowed to be stretched in between the heavier brane and its image whenever the corresponding $\mathbf{Z}_{2}$ flux of the form which couples to it electrically is non-trivial does not apply here. It is not clear how the picture generalizes.

Similar statements hold for D3 branes stretching between NS5 branes in the presence of the different types of $O N 5_{B}$ planes.

\subsection{Orientifold webs}

Since we identified $(p, q)$ O1 lines and $(p, q)$ O5 planes one may wonder whether $(p, q)$ webs of orientifolds are possible. We would like to show that those may be possible in some special cases, but in general they do not make sense.

Let us consider the basic junction at $\tau=i$, where $\tau$ is the complex scalar of Type IIB. We have a horizontal $(0,1)$ O1 meeting a vertical $(1,0)$ OF1 and a $(1,1)$ O line which leaves the junction at 45 degrees. Each orientifold plane requires a $\mathbf{Z}_{2}$ projection. If we ignore the orientation reversal and consider only the spatial inversion we see that these 3 reflections generate a group of order 8 , isomorphic to $D_{4}$, the dihedral group of 4 elements. It is not clear how do the different orientation reversals combine.

However, if there are two $\mathrm{O}$ lines at an irrational relative angle $\alpha$ (measured in radians $/ 2 \pi$ ) then a composition of both reflections gives a rotation by $2 \alpha$. Since we assumed $\alpha$ irrational, then this element generates an infinite group of identifications on the plane, generating a non-discrete image set from a single point. Thus we cannot hope to have an ordinary orbifold/orientifold, and this is the case for generic $\tau$.

\section{Acknowledgements}

We thank O. Bergman, E. Gimon, S. Gukov, E. Shustin, C. Vafa, E. Witten and A. Zaffaroni for enjoyable discussions.

A.H. would like to thank the Institute for Theoretical Physics at Santa Barbara and Tel-Aviv University for their kind hospitality while various stages of this work were completed. B.K. would like to thank J. Sonnenschein, S. Yankielowicz and the rest of the group at Tel Aviv. 
A. H. is partially supported by the National Science Foundation under grant no. PHY94-07194, by the DOE under grant no. DE-FC02-94ER40818, by an A. P. Sloan Foundation Fellowship and by a DOE OJI award. The research of BK was supported in part by the US-Israeli Binational Science Foundation, the German-Israeli Foundation for Scientific Research (GIF), and the Israel Science Foundation.

\section{A. Appendix - Cohomology of RP}

We distinguish between two kinds of cohomologies. The twisted ones, denoted by $\tilde{H}$ classify "twisted" forms. These are forms which reverse sign under the projection (such forms are not related to a twisted sector). The ordinary "untwisted" cohomologies are denoted just by $H$ and are appropriate to classify forms which do not change sign under the projection.

The integral cohomologies of $\mathbf{R P}^{\mathbf{n}}$ are

$$
\begin{aligned}
& H^{q}= \begin{cases}\mathbf{Z}_{2} & q \text { even but } q \neq 0 \\
\mathbf{Z} & q=0, \text { and for odd } \mathrm{n} \quad q=n \\
0 & \text { otherwise }\end{cases} \\
& \tilde{H}^{q}= \begin{cases}\mathbf{Z}_{2} & q \text { odd } \\
\mathbf{Z} & \text { for even } \mathrm{n} q=n \\
0 & \text { otherwise }\end{cases}
\end{aligned}
$$

These results can be easily deduced from the chain complexes

$$
\begin{gathered}
\leftarrow C^{2}=\mathbf{Z} \stackrel{\times 2}{\longleftarrow} C^{1}=\mathbf{Z} \stackrel{0}{\longleftarrow} C^{0}=\mathbf{Z} \leftarrow 0 \\
\stackrel{\times 2}{\longleftarrow} C^{2 m+1}=\mathbf{Z} \stackrel{0}{\longleftarrow} C^{2 m}=\mathbf{Z} \stackrel{\times 2}{\longleftarrow} \ldots \\
0 \leftarrow C^{n}=\mathbf{Z} \leftarrow \ldots
\end{gathered}
$$

and

$$
\begin{gathered}
\leftarrow \tilde{C}^{2}=\mathbf{Z} \stackrel{0}{\longleftarrow} \tilde{C}^{1}=\mathbf{Z} \stackrel{\times 2}{\longleftarrow} \tilde{C}^{0}=\mathbf{Z} \leftarrow 0 \\
\stackrel{0}{\longleftarrow} \tilde{C}^{2 m+1}=\mathbf{Z} \stackrel{\times 2}{\longleftarrow} \tilde{C}^{2 m}=\mathbf{Z} \stackrel{0}{\longleftarrow} \ldots \\
0 \leftarrow \tilde{C}^{n}=\mathbf{Z} \leftarrow \ldots
\end{gathered}
$$

where $C^{q}$ are the q-cochains and $\tilde{C}^{q}$ are the twisted q-cochains.

For completeness we list the integral homology groups as well

$$
\begin{aligned}
& H_{q}= \begin{cases}\mathbf{Z}_{2} & q \text { odd but } q \neq n \\
\mathbf{Z} & q=0, \text { and for odd } \mathrm{n} q=n \\
0 & \text { otherwise }\end{cases} \\
& \tilde{H}_{q}= \begin{cases}\mathbf{Z}_{2} & q \text { even } q \neq n \\
\mathbf{Z} & \text { for even } \mathrm{n} q=n \\
0 & \text { otherwise }\end{cases}
\end{aligned}
$$


For odd $n \mathbf{R P}^{\mathbf{n}}$ is orientable and Poincare duality holds

$$
H_{i}=H^{n-i}, \quad \tilde{H}_{i}=\tilde{H}^{n-i}
$$

For even $n$, on the other hand

$$
H_{i}=\tilde{H}^{n-i}, \quad \tilde{H}_{i}=H^{n-i}
$$

\section{References}

[1] A. Sagnotti, "Open Strings And Their Symmetry Groups," ROM2F-87/25 Talk presented at the Cargese Summer Institute on Non-Perturbative Methods in Field Theory, Cargese, Italy, Jul 16-30, $198 \%$.

[2] P. Hořava, "Strings On World Sheet Orbifolds," Nucl. Phys. B327, 461 (1989). SPIRES

[3] E. G. Gimon and J. Polchinski, "Consistency Conditions for Orientifolds and DManifolds," Phys. Rev. D54, 1667 (1996) [hep-th/9601038]. SPIRES

[4] L. Jarv, C. V. Johnson, "Orientifolds, M-Theory, and the ABCD's of the Enhancon," hep-th/0002244. hep-th/0002244.

[5] Z. Kakushadze, "Geometry of orientifolds with NS-NS B-flux," hep-th/0001212. SPIRES

[6] A. M. Uranga, "Comments on non-supersymmetric orientifolds at strong coupling," hepth/9912145. SPIRES

[7] C. Vafa, "Modular Invariance And Discrete Torsion On Orbifolds," Nucl. Phys. B273, 592 (1986). SPIRES

[8] C. Vafa and E. Witten, "On orbifolds with discrete torsion," J. Geom. Phys. 15, 189 (1995) [hep-th/9409188]. SPIRES

[9] R. Dijkgraaf, "Discrete torsion and symmetric products," hep-th/9912101. SPIRES

[10] J. Gomis, "D-branes on orbifolds with discrete torsion and topological obstruction," hep-th/0001200. SPIRES

[11] M. Klein and R. Rabadan, "Orientifolds with discrete torsion," hep-th/0002103. SPIRES

[12] C. Angelantonj and R. Blumenhagen, "Discrete deformations in Type I vacua," hepth/9911190. SPIRES

[13] A. Hanany and E. Witten, "Type IIB superstrings, BPS monopoles, and threedimensional gauge dynamics," Nucl. Phys. B492, 152 (1997) [hep-th/9611230]. SPIRES 
[14] O. Bergman, A. Hanany, A. Karch and B. Kol, "Branes and supersymmetry breaking in 3D gauge theories," JHEP 9910, 036 (1999) [hep-th/9908075]. SPIRES A. Armoni and B. Kol, "Non-supersymmetric large N gauge theories from type 0 brane configurations," JHEP 9907, 011 (1999) [hep-th/9906081]. SPIRES

[15] K. Dasgupta and S. Mukhi, "Orbifolds of M-theory," Nucl. Phys. B465, 399 (1996) [hep-th/9512196]. SPIRES

[16] A. Hanany, B. Kol and A. Rajaraman, "Orientifold points in M theory," JHEP 9910, 027 (1999) [hep-th/9909028]. SPIRES

[17] S. Sethi, "A relation between $N=8$ gauge theories in three dimensions," JHEP 11, 003 (1998) hep-th/9809162. SPIRES

[18] M. Berkooz and A. Kapustin, "New IR dualities in supersymmetric gauge theory in three dimensions," JHEP 02, 009 (1999) hep-th/9810257. SPIRES

[19] E. Witten, "Five-branes and M-theory on an orbifold," Nucl. Phys. B463, 383 (1996) [hep-th/9512219]. SPIRES

[20] K. Hori, "Consistency condition for fivebrane in M-theory on R**5/Z(2) orbifold," Nucl. Phys. B539, 35 (1999) hep-th/9805141. SPIRES

[21] E.G. Gimon, "On the M-theory interpretation of orientifold planes," hep-th/9806226. SPIRES

[22] J. H. Schwarz, "An SL(2,Z) multiplet of Type IIB superstrings," Phys. Lett. B360, 13 (1995) [hep-th/9508143]. SPIRESP. S. Aspinwall, "Some relationships between dualities in string theory," Nucl. Phys. Proc. Suppl. 46, 30 (1996) [hep-th/9508154]. SPIRES

[23] B. Kol, "On 6d "gauge" theories with irrational theta angle," JHEP 9911, 017 (1999) [hep-th/9711017]. SPIRES B. Kol, "5d field theories and M theory," JHEP 9911, 026 (1999) [hep-th/9705031]. SPIRES

[24] G. Moore and E. Witten, "Self-duality, Ramond-Ramond fields, and K-theory," hepth/9912279. SPIRES

[25] R. Minasian and G. Moore, "K-theory and Ramond-Ramond charge," JHEP 9711, 002 (1997) [hep-th/9710230]. SPIRES

[26] E. Witten, "D-branes and K-theory," JHEP 9812, 019 (1998) [hep-th/9810188]. SPIRES

[27] P. Hořava, "Type IIA D-branes, K-theory, and matrix theory," Adv. Theor. Math. Phys. 2, 1373 (1999) [hep-th/9812135]. SPIRES 
[28] O. Bergman, E. G. Gimon and P. Hořava, "Brane transfer operations and T-duality of non-BPS states," JHEP 9904, 010 (1999) [hep-th/9902160]. SPIRES

[29] S. Gukov, "K-theory, reality, and orientifolds," hep-th/9901042. SPIRES

[30] K. Hori, "D-branes, T-duality, and index theory," hep-th/9902102. SPIRES

[31] A. Dabholkar and J. Park, "Strings on Orientifolds," Nucl. Phys. B477, 701 (1996) [hep-th/9604178]. SPIRES

[32] A. M. Uranga, "Towards mass deformed $\mathrm{N}=4 \mathrm{SO}(\mathrm{n})$ and $\mathrm{Sp}(\mathrm{k})$ theories from brane configurations," Nucl. Phys. B526, 241 (1998) [hep-th/9803054]. SPIRES

[33] E. Witten, "Baryons and branes in anti de Sitter space," JHEP 9807, 006 (1998) [hepth/9805112]. SPIRES

[34] E. Witten, "New *gauge* theories in six dimensions," JHEP 9801, 001 (1998) [hepth/9710065]. SPIRES

[35] J. de Boer, R. Dijkgraaf, A. Keurentjes, J. Morgan, D. Morrison, S. Sethi as reported in the talk of R. Dijkgraaf at "String Theory at The Millennium" conference http://quark.theory.caltech.edu/people/rahmfeld/Dijkgraaf/fs1.htm], p.19-26.

[36] A. Hanany and A. Zaffaroni, "Issues on orientifolds: On the brane construction of gauge theories with SO(2n) global symmetry," JHEP 9907, 009 (1999) [hep-th/9903242]. SPIRES

[37] N. Evans, C. V. Johnson and A. D. Shapere, "Orientifolds, branes, and duality of 4D gauge theories," Nucl. Phys. B505, 251 (1997) [hep-th/9703210]. SPIRES

[38] S. Elitzur, A. Giveon, D. Kutasov and D. Tsabar, "Branes, orientifolds and chiral gauge theories," Nucl. Phys. B524, 251 (1998) [hep-th/9801020]. SPIRES

[39] S. Mukhi and N. V. Suryanarayana, "Gravitational couplings, orientifolds and M-planes," JHEP 9909, 017 (1999) [hep-th/9907215]. SPIRES

[40] I. Brunner, A. Hanany, A. Karch and D. Lust, "Brane dynamics and chiral non-chiral transitions," Nucl. Phys. B528, 197 (1998) [hep-th/9801017].

[41] P. Hořava and E. Witten, "Eleven-Dimensional Supergravity on a Manifold with Boundary," Nucl. Phys. B475, 94 (1996) [hep-th/9603142]. SPIRES

[42] E. Witten, "On flux quantization in M-theory and the effective action," J. Geom. Phys. 22, 1 (1997) hep-th/9609122. SPIRES

[43] C. Ahn, H. Kim and H. S. Yang, "SO(2N)(0,2) SCFT and M theory on AdS(7) x RP(4)," Phys. Rev. D59, 106002 (1999) [hep-th/9808182]. SPIRES 
[44] C. Ahn, H. Kim, B. Lee and H. S. Yang, "N=8 SCFT and M theory on AdS(4) x RP(7)," Phys. Rev. D61, 066002 (2000) [hep-th/9811010]. SPIRES

[45] O. Aharony, J. Sonnenschein and S. Yankielowicz, "Interactions of strings and D-branes from M theory," Nucl. Phys. B474, 309 (1996) [hep-th/9603009]. SPIRES

[46] N. Kim and S. Rey, "M(atrix) theory on T(9)/Z(2) orbifold and twisted zero-branes," hep-th/9710245. SPIRES

[47] V. G. Kac and A. V. Smilga, "Normalized vacuum states in N $=4$ supersymmetric Yang-Mills quantum mechanics with any gauge group," hep-th/9908096. SPIRES

[48] K. Dasgupta and S. Mukhi, "A note on low-dimensional string compactifications," Phys. Lett. B398, 285 (1997) [hep-th/9612188]. SPIRES

[49] L. Dixon, J. A. Harvey, C. Vafa and E. Witten, "Strings On Orbifolds," Nucl. Phys. B261, 678 (1985). L. Dixon, J. A. Harvey, C. Vafa and E. Witten, "Strings On Orbifolds. 2," Nucl. Phys. B274, 285 (1986).

[50] A. Sen, "Duality and Orbifolds," Nucl. Phys. B474, 361 (1996) [hep-th/9604070]. SPIRES

[51] A. Sen, "Stable non-BPS bound states of BPS D-branes," JHEP 9808, 010 (1998) [hepth/9805019]. SPIRES 